\title{
Contextual Information Drives the Reconsolidation-Dependent Updating of Retrieved Fear Memories
}

\author{
Timothy J Jarome ${ }^{1,2,4}$, Nicole C Ferrara ${ }^{1,4}$, Janine L Kwapis ${ }^{1,3}$ and Fred J Helmstetter, ${ }^{*, 1}$ \\ 'Department of Psychology, University of Wisconsin-Milwaukee, Milwaukee, WI, USA
}

\begin{abstract}
Stored memories enter a temporary state of vulnerability following retrieval known as 'reconsolidation', a process that can allow memories to be modified to incorporate new information. Although reconsolidation has become an attractive target for treatment of memories related to traumatic past experiences, we still do not know what new information triggers the updating of retrieved memories. Here, we used biochemical markers of synaptic plasticity in combination with a novel behavioral procedure to determine what was learned during memory reconsolidation under normal retrieval conditions. We eliminated new information during retrieval by manipulating animals' training experience and measured changes in proteasome activity and GluR2 expression in the amygdala, two established markers of fear memory lability and reconsolidation. We found that eliminating new contextual information during the retrieval of memories for predictable and unpredictable fear associations prevented changes in proteasome activity and glutamate receptor expression in the amygdala, indicating that this new information drives the reconsolidation of both predictable and unpredictable fear associations on retrieval. Consistent with this, eliminating new contextual information prior to retrieval prevented the memory-impairing effects of protein synthesis inhibitors following retrieval. These results indicate that under normal conditions, reconsolidation updates memories by incorporating new contextual information into the memory trace. Collectively, these results suggest that controlling contextual information present during retrieval may be a useful strategy for improving reconsolidation-based treatments of traumatic memories associated with anxiety disorders such as post-traumatic stress disorder.

Neuropsychopharmacology (2015) 40, 3044-3052; doi:I0.1038/npp.20I5.161; published online 8 July 2015
\end{abstract}

\section{INTRODUCTION}

Traumatic memories associated with a variety of anxiety disorders such as post-traumatic stress disorder are often difficult to treat therapeutically. One of the more traditional approaches is therapy based on extinction training, which results in a gradual reduction in fear responses to cues associated with the trauma. However, this is not a permanent reduction, as fear responses to these cues will often return with time (Pavlov, 1927; Parsons and Ressler, 2013). For this reason new methods are needed for the treatment of fear responses associated with traumatic memories. One potential method is reconsolidation-based treatment.

Reconsolidation is a unique, temporally limited process in which retrieval of a memory initiates a transient period during which new information can be incorporated into the

* Correspondence: Professor FJ Helmstetter, Department of Psychology, Garland Hall, University of Wisconsin-Milwaukee, P.O. Box 413, Milwaukee, WI 5320I, USA, Tel: +4I4 229-4903, Fax: +4I4 2295219 , E-mail: fjh@uwm.edu

${ }^{2}$ Present address: Department of Neurobiology, University of Alabama at Birmingham, Birmingham, AL, USA.

${ }^{3}$ Present address: Department of Neurobiology and Behavior, University of California Irvine, Irvine, CA, USA.

${ }^{4}$ These authors contributed equally to this work.

Received 2 December 2014; revised 12 May 2015; accepted 4 June 2015; accepted article preview online II June 2015 memory trace for a previously learned task or association (Lee, 2008; Alberini and Chen, 2012; Finnie and Nader, 2012; Alberini and Ledoux, 2013). This reconsolidation process requires de novo protein synthesis in neurons (Nader et al, 2000; Parsons et al, 2006) and is thought to be initiated by increased ubiquitin-proteasome-mediated protein degradation and changes in the expression of GluR2 containing AMPA receptors at synapses (Lee et al, 2008; Jarome et al, 2011; Rao-Ruiz et al, 2011; Hong et al, 2013). For example, inhibiting proteasome activity or the exchange of GluR2 containing for GluR2 lacking AMPA receptors in the amygdala can prevent the reconsolidation process from occurring, suggesting that the reconsolidation process primarily relies on increased protein degradation and AMPA receptor subunit exchange.

The reconsolidation of memories for cues associated with traumatic events has often been studied using Pavlovian fear conditioning (Nader et al, 2000). In this paradigm, a neutral conditioned stimulus (CS) is paired with a noxious unconditioned stimulus (UCS). Once this association has been established, the CS can elicit conditional responses. Different auditory, olfactory and visual stimuli can act as a CS during fear conditioning and, in most cases, the context or environment that these cues occur in also becomes associated with the UCS. In general, the amygdala is required for the formation of fear memories learned through Pavlovian fear conditioning and for their reconsolidation after retrieval. 
The memory reconsolidation process has become an attractive target for treatment of traumatic memories associated with anxiety disorders (Alberini and Ledoux, 2013). Indeed, evidence from this paradigm now suggests that manipulating new information during retrieval of an established memory can not only result in changes to the original memory but also the persistent attenuation of fear responses to cues associated with aversive events (Monfils et al, 2009; Clem and Huganir, 2010; Schiller et al, 2010; Rao-Ruiz et al, 2011). However, while targeting reconsolidation can serve a therapeutic function in the treatment of traumatic memories, it also can promote both the persistence of fear and the resistance of these memories to extinction training, which occurs following a single presentation of the cue (Inda et al, 2011). This strengthening of memory as a result of the reconsolidation process supports the theory that reconsolidation likely updates a memory each time it is retrieved (Tronson et al, 2006; Jarome et al, 2012). However, what new information is being incorporated into the memory trace through the reconsolidation process is unknown. A more detailed understanding of the novel information added to the memory trace during reconsolidation is critical for the potential use of this process as a therapeutic target in the treatment of pathological fear and traumatic memories.

A currently popular view is that reconsolidation and updating of memories is driven by 'prediction error' or a change in the relationship between the specific cues present during the aversive event and their ability to predict the occurrence of that event at later times. Consistent with this, changing the relationship between the cue and its aversive outcome can control the ability of a retrieved memory to undergo reconsolidation in both rodents and human subjects (Diaz-Mataix et al, 2013; Sevenster et al, 2013). However, these studies presented the cue and the aversive stimulus during the retrieval session, which is not common in normal reconsolidation procedures where the CS is often presented alone. Furthermore, it is unknown if prediction error can explain why a memory updates under normal conditions when only the predictive signal is present. In addition, the presentation of the cue by itself during retrieval can result in the reconsolidation-dependent strengthening of memory and prevent the time-dependent forgetting of a memory (Inda et al, 2011), suggesting that the aversive UCS does not need to be present for the strengthening of the memory or the persistence of fear over time. This suggests that some other information may be present during retrieval that drives the updating of the memory trace. In the present study, we wanted to determine what new information triggers memory updating during Pavlovian fear conditioning by systematically manipulating the animals' training experience so that no new information would be present during retrieval. Surprisingly, we found that new contextual information controlled the reconsolidation of retrieved memories.

\section{MATERIALS AND METHODS}

\section{Subjects}

Male Long-Evans rats weighing between 300 and $350 \mathrm{~g}$ at time of arrival were obtained from Harlan (Madison, WI). All animals were housed individually in opaque plastic cages with free access to water and rat chow. The colony room was maintained under a 14:10-h light/dark cycle. Experiments took place during the light portion of the cycle. All procedures were approved by the University of WisconsinMilwaukee Institutional Animal Care and Use Committee and conducted within the ethical guidelines of the National Institutes of Health.

\section{Apparatus}

Auditory fear conditioning was conducted in a set of four Plexiglas and stainless-steel observation chambers $(21 \times 28 \times 21 \mathrm{~cm}$; Context A) housed in sound-attenuating chambers. The floor was comprised of 18 stainless-steel bars $5 \mathrm{~mm}$ in diameter, spaced $12 \mathrm{~mm}$ apart and connected to a shock generator. Ventilation fans produced $62-64 \mathrm{~dB}$ of background noise. Each chamber was equipped with a speaker centered in the middle of one end of the chamber. Before testing of each animal, Context A was cleaned with a $5 \%$ ammonium hydroxide solution. The retrieval chambers (Context B) had several distinct features relative to Context A, including infrared lighting, textured floors and a 5\% acetic acid smell (Gilmartin et al, 2013a). Context C was a Med Associates conditioning chamber $(30.5 \times 24.1 \times 29.2 \mathrm{~cm})$ housed in a sound attenuating outer chamber and illuminated with a white incandescent house light (Gilmartin et al, 2013b). Context C was wiped with ethanol prior to use.

\section{Surgery and Infusion Procedures}

Animals were anesthetized with $2-4 \%$ isoflurane in $100 \% \mathrm{O}_{2}$ and implanted with bilateral stainless-steel 26-gauge cannulae aimed at the basolateral nucleus of the amygdala (AP - 3.0 $\mathrm{mm}, \mathrm{ML} \pm 5.0 \mathrm{~mm}, \mathrm{DV}-7.2 \mathrm{~mm}$ ) using stereotaxic coordinates relative to bregma. Cannulae were secured to the skull with stainless-steel screws, superglue and dental acrylic. Rats were given a recovery period of at least 7 days before behavioral testing. Rats received bilateral infusions into the amygdala. The total volume of the infusion $(0.5 \mu \mathrm{l} / \mathrm{side})$ was given over $60 \mathrm{~s}$, and the injection cannula remained in place an additional $90 \mathrm{~s}$ to ensure diffusion away from the injector tip. The injection cannulae were cut to extend $\sim 0.5 \mathrm{~mm}$ beyond the guide cannula. Rats were returned to their home cages after infusions. Anisomycin (ANI, $125 \mu \mathrm{g} / \mu \mathrm{l}$; Tocris) was dissolved in $\mathrm{HCl}$ and diluted with artificial CSF. The $\mathrm{pH}$ was $\sim 7.3$.

\section{Behavioral Procedures}

All animals were handled for 3 days in the animal colony and acclimated for 3 days to the transport (protein assays) or restraint procedures (microinfusions) prior to the start of behavioral training. For auditory fear conditioning, animals were placed into context $\mathrm{A}$, and after a 6-min baseline period received four white noise $(72 \mathrm{~dB}, 10 \mathrm{~s}$ shock $(1.0 \mathrm{~mA} / 1 \mathrm{~s})$ pairings with a 90 -s intertrial interval. After a 4 -min postshock period, the animals were removed from the chambers. The next day, animals were placed into novel context $B$ and after a 90-s baseline, presented with a 30-s white noise presentation in the absence of shock to reactivate the memory. Animals were then removed from the chamber and returned to their homecages. For 50\% reinforcement auditory fear conditioning, animals were placed into context A, and after a 6-min baseline received four white noise 
(72 dB, $10 \mathrm{~s})$ - shock (1.0 mA/1 s) pairings (WN-SK) and four white noise $(72 \mathrm{~dB}, 10 \mathrm{~s})$ only presentations (WN) with a 90-s intertrial interval. The $\mathrm{WN}-\mathrm{SK}$ and $\mathrm{WN}$ presentations were given in a pseudorandom order (WN-SK, WN, WN-SK, WNSK, WN, WN, WN-SK, WN). After a 4-min post-shock period, the animals were removed from the chambers. In cases where animals received 'retrieval pre-exposure' the day prior to training, they were placed in novel context B and after a 90$s$ baseline presented with a 30 -s white noise presentation in the absence of shock. In control experiments, animals were placed in context $\mathrm{B}$ for $2 \mathrm{~min}$ without a white noise presentation or placed in Context $\mathrm{C}$ for 90 -s followed by a 30 -s white noise presentation. Testing occurred $24 \mathrm{~h}$ after retrieval and consisted of placing the animals back in context $\mathrm{B}$ and after a 60 -s baseline, eight 30 -s white noise presentations were given (60-s ITI). As behavioral performance declines after the third CS presentation, the first three CS presentations were used for subsequent analyses. This is consistent with our previous work demonstrating that the animals start to undergo extinction after the third CS presentation during the testing session (Kwapis et al, 2011). In all cases, animals were always returned to the animal colony following the completion of pre-exposure, acquisition, retrieval and testing sessions.

\section{Tissue Collection}

Crude synaptosomal membrane fractions were collected as described previously (Jarome et al, 2011, 2012). Animals were overdosed on isoflurane, and the brain rapidly removed and immediately frozen on dry ice. Amygdala and dorsal hippocampus tissue was then dissected out by blocking the brain in a rat brain matrix (Harvard Apparatus, Holliston, MA). Tissue samples were homogenized in TEVP with $320 \mathrm{mM}$ sucrose plus Roche protease inhibitor complete tablet and centrifuged at $1000 \times g$ for $10 \mathrm{~min}$ at $4{ }^{\circ} \mathrm{C}$. The supernatant was collected and centrifuged at $10000 \times g$ for $10 \mathrm{~min}$ at $4{ }^{\circ} \mathrm{C}$. The resulting pellet was denatured in lysis buffer $(50 \mathrm{mM}$ Tris- $\mathrm{HCl}, 150 \mathrm{mM} \mathrm{NaCl}, 1 \mathrm{mM}$ EDTA, $1 \mathrm{mM}$ $\mathrm{NaF}, 10 \mathrm{ml} 10 \%$ SDS, $1 \mathrm{mM}$ sodium orthovanadate and Roche protease inhibitor complete tablet) and centrifuged at $15000 \times g$ for $5 \mathrm{~min}$ at $4{ }^{\circ} \mathrm{C}$. The supernatant was collected and measured using a Bradford protein assay kit.

\section{S Proteasome Activity Assay}

Proteasome activity assays were performed as described previously (Jarome et al, 2013b). Samples (10 $\mu \mathrm{g})$ were diluted in $\mathrm{DDH}_{2} \mathrm{O}$ and mixed with reaction buffer $(250 \mathrm{mM}$ HEPES, pH 7.5, $5 \mathrm{mM}$ EDTA, 0.5\% NP-40, 0.01\% SDS, $5 \mathrm{mM}$ ATP). Fluorogenic peptides Suc-LLVY-AMC (Millipore) or Bz-VGR-AMC (Enzo Life Sciences) were added to the samples to assess proteasome chymotrypsin-like or trypsin-like activities, respectively $(10 \mu \mathrm{M})$. The reaction was incubated at $37^{\circ} \mathrm{C}$ for $30 \mathrm{~min}$ (Bz-VGR-AMC) or $2 \mathrm{~h}$ (Suc-LLVY-AMC) and fluorescence monitored at 360 (excitation)/460 (emission) on a monochromatic plate reader (Synergy H1; Biotek). Protein-free blanks were used and an AMC standard curve was produced.

\section{Western Blotting}

Samples $(10 \mu \mathrm{g})$ were loaded on $7.5 \%$ TGX gels, ran through SDS-PAGE and transferred using a Turbo Transfer System (Biorad). Membranes were incubated in 3\% milk in TBS $+0.1 \%$ Tween-20 (blocking buffer) for $1 \mathrm{~h}$ at room temperature, followed by overnight incubation in antibody in 3\% BSA in TBS+0.1\% Tween-20. Membranes were then washed and incubated in secondary antibody (1:20,000; Millipore for goat anti-rabbit, Santa Cruz for goat anti-mouse) in blocking buffer for $60 \mathrm{~min}$. Following a final wash, membranes were incubated in enhanced chemiluminescence substrate (SuperSignal West Dura, Thermo) for $5 \mathrm{~min}$ and images developed using a CCD-camera based system (GBOX Chemi XT-4; Syngene) and analyzed using GeneTools software. Primary antibodies included Actin (1:1000; Cell Signaling), GluR1 (1:1000; Millipore) and GluR2 (1:1000; NeuroMab).

\section{Conditioned Fear Responses}

The freezing behavior of each rat was recorded on digital video and the amount of movement determined by frame-by-frame changes in pixels using FreezeScan 1.0 software (CleverSys, Reston, VA). The automatic scoring parameters are chosen such that the scored activity matches hand-scoring methods previously used in our laboratory to measure freezing.

\section{Statistical Analyses}

For quantitative protein assays, mean pixel density was calculated for each sample and taken as a percentage of the control group. For proteasome activity assays, each raw fluorescence reading was standardized to the generated AMC standard curve for that plate and taken as a percentage of the control group. Statistical outliers were defined as those that fell two or more SDs above or below the group mean and were determined using the outlier function in Prism 6 software (Graphpad). Data were analyzed using analysis of variance and Fisher least significant difference post hoc tests unless otherwise indicated.

\section{RESULTS}

In these experiments, rats were trained with auditory fear conditioning in which a cue predicts an aversive footshock. Retrieval of this memory results in an increased need for protein synthesis in the amygdala (Parsons et al, 2006; Jarome et al, 2011, 2012), a hallmark component of the reconsolidation process (Nader et al, 2000). First, we established that this protocol results in 'destabilization' or a temporary lability of the memory trace by measuring postretrieval levels of proteasome activity and GluR2 (Figure 1a), two well-established markers of memory destabilization and reconsolidation (Lee et al, 2008; Lee, 2008; Clem and Huganir, 2010; Jarome et al, 2011; Rao-Ruiz et al, 2011; Hong et al, 2013). Using an in vitro proteasome activity assay and western blotting, we found that memory retrieval increased proteasome activity $\left(F_{(2,26)}=4.463, \quad P<0.05\right.$; Figure 1b) and decreased GluR1 $\left(F_{(2,25)}=3.287, P=0.054\right)$ and GluR2 $\left(F_{(2,26)}=3.459, P<0.05\right)$ levels (Figure $\left.1 \mathrm{c}\right)$ at amygdala synapses without altering $\beta$-actin expression 
a

Day 1 4 CS-UCS

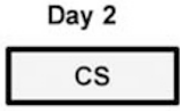

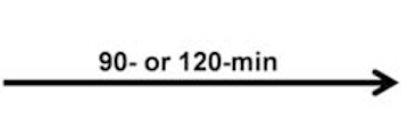

Amygdala
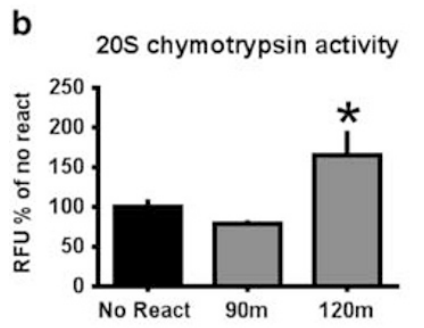

c

GluR1
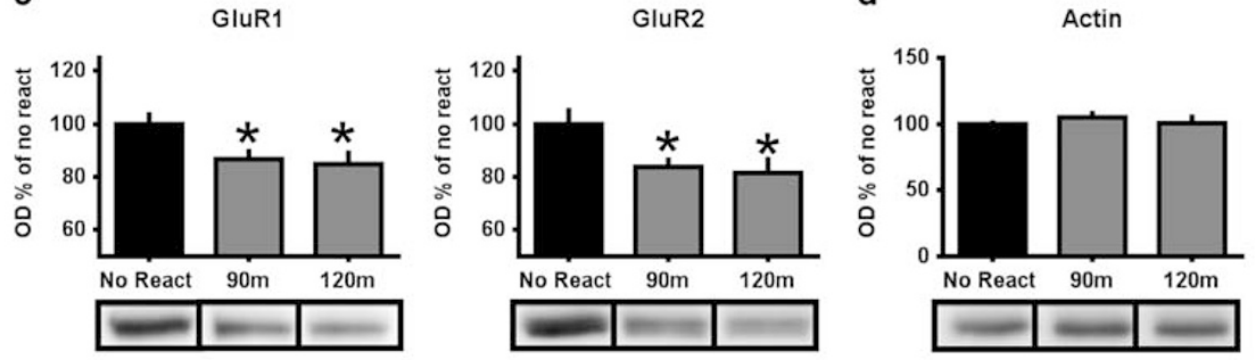
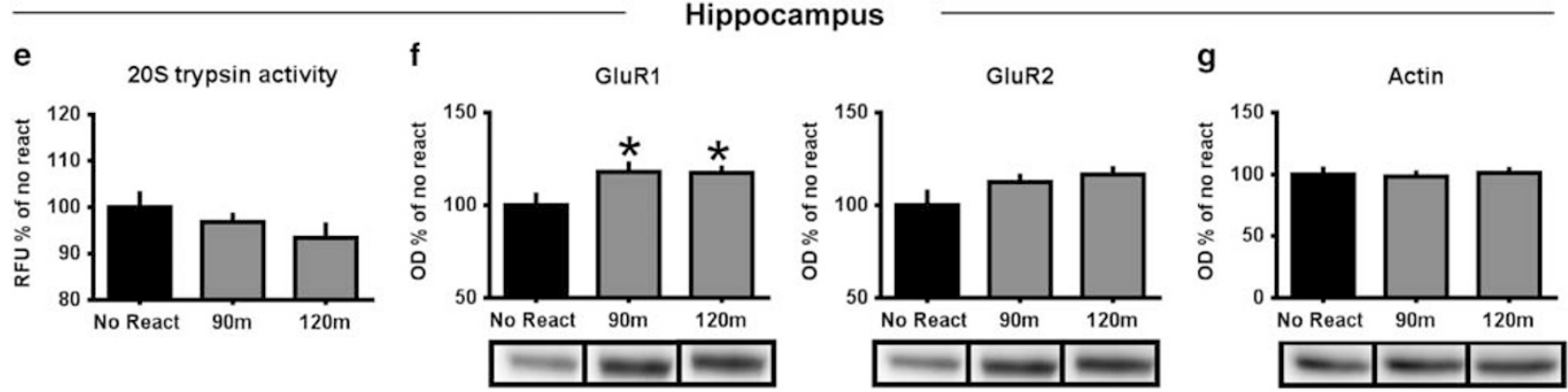

Figure I Memory retrieval increases in vtiro proteasome activity and decreases GluR2 in the amygdala and increases GluRI in the hippocampus. (a) Rats were exposed to several pairings of an auditory cue with a footshock and amygdala tissue collected 90 or 120 min later ( $n=10-11$ per group). (b) Proteasome activity was increased 120 min after retrieval. (c) GluRI and GluR2 levels were decreased I20 min after retrieval with no change in $\beta$-actin expression (d). Memory retrieval did not alter proteasome activity (e) but did increase GluRI expression (f) in the hippocampus. There were no changes in $\beta$-actin expression in the hippocampus $(\mathrm{g})$. $* P<0.05$ from no retrieval.

a

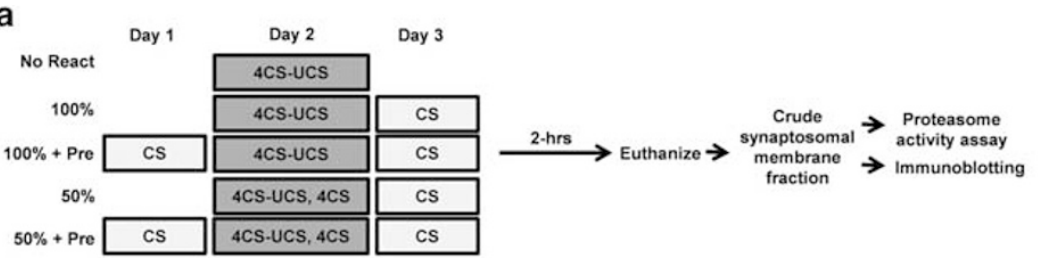

b

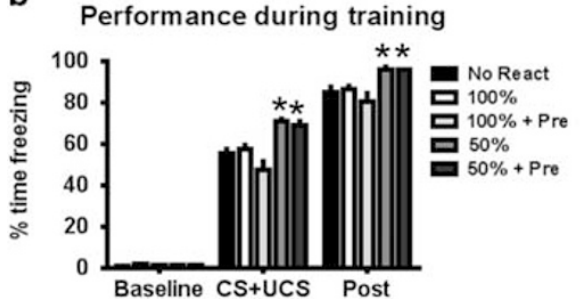

C Retention during CS retrieval

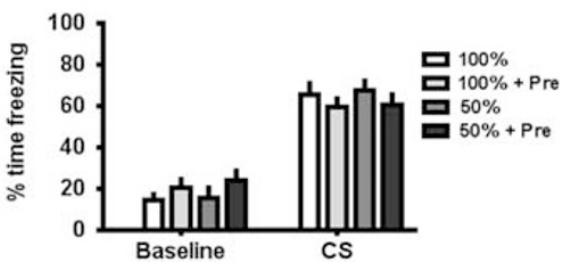

Figure 2 Partial reinforcement training and retrieval pre-exposure did not alter retention to the auditory cue during retrieval. (a) Rats were trained to I00\% or $50 \%$ reinforcement auditory fear conditioning with or without 'retrieval pre-exposure' the day before training. The groups that received partial reinforcement froze significantly more during the training session (b), but all groups showed equal retention to the auditory cue during the retrieval session (c). $* P<0.05$ from no retrieval.

$\left(F_{(2,29)}=0.267, P=0.768\right.$; Figure $\left.1 \mathrm{~d}\right)$. This confirmed that the memory triggered by the auditory cue underwent reconsolidation in the amygdala following retrieval.

Although the reconsolidation of auditory fear memories is largely regulated by the amygdala, the aversive auditory cue is being presented in a new environment during retrieval.
This leads to the possibility that other brain regions may be recruited during the reconsolidation process. To test this, we examined changes in proteasome activity and AMPA receptor expression in the dorsal hippocampus, a brain region that is important in the coding of contextual information but is not required for the initial formation 
a

20 S chymotrypsin activity in the amygdala

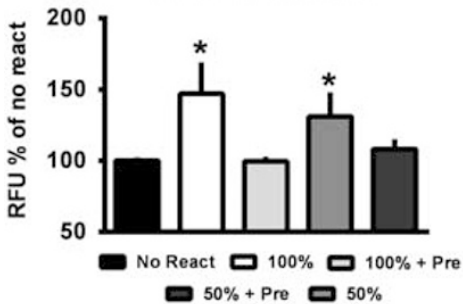

20 S trypsin activity in the hippocampus

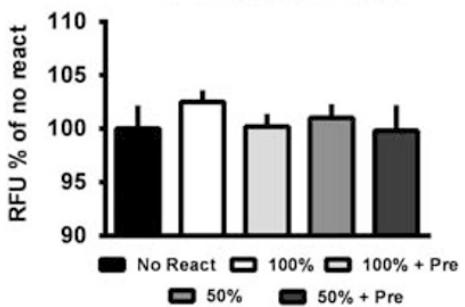

b

GluR1 expression in the amygdala

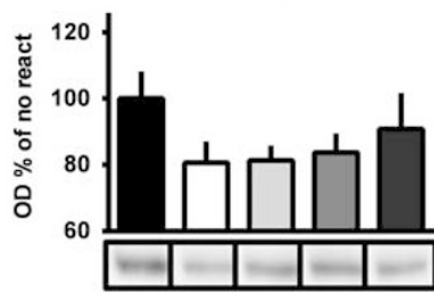

d

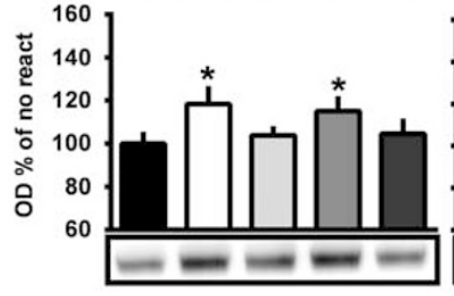

GluR2 expression in the amygdala
Actin expression

in the amygdala

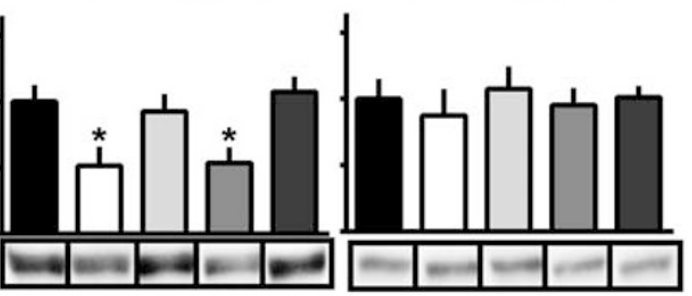

GluR2 expression in the hippocampus

Actin expression in the hippocampus

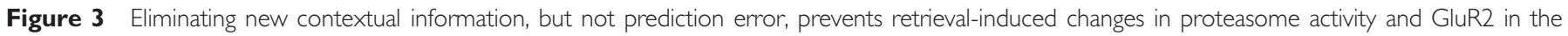

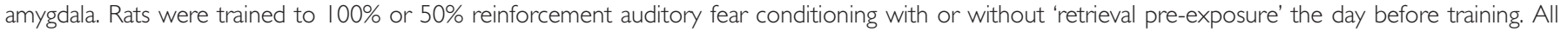
animals received a retrieval on day 3 and amygdala tissue collected 120 min later $(n=12-13$ per group). (a and b) Eliminating new contextual information prevented increases in proteasome activity (a) and decreases in GluR2 in the amygdala without altering GluR I or $\beta$-actin expression (b). Eliminating contextual

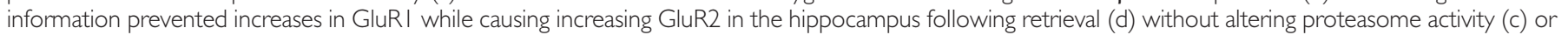
$\beta$-actin. $* P<0.05$.

and consolidation of memory for auditory fear associations. Surprisingly, although we did not see changes in proteasome activity $\left(F_{(2,29)}=1.193, P=0.318\right.$; Figure $\left.1 \mathrm{e}\right)$, we did see increases in GluR1 $\left(F_{(2,26)}=3.194, P=0.058\right)$ but not GluR2 $\left(F_{(2,26)}=2.007, P=0.155\right.$; Figure 1f) or $\beta$-actin $\left(F_{(2,27)}=0.098\right.$, $P=0.907$; Figure 1g) expression at hippocampal synapses following retrieval. This suggests that the hippocampus may be involved in the reconsolidation of auditory fear memories, perhaps through incorporation of new contextual information.

We next tested what new information was initiating the reconsolidation process by manipulating the animals' training experience so that no new information would be present during the retrieval session. To eliminate the potential sources of new information during retrieval, we manipulated the ability of the auditory cue to accurately predict shock during the training experience (Figure 2a). We trained animals with either $100 \%$ reinforcement (predictable) where the auditory cue was always followed by shock or $50 \%$ reinforcement (unpredictable) where the shock was presented on only half of the auditory cue trials. This type of training results in conditions in which the auditory cue is a perfect predictor of the shock (predictable) or the auditory cannot accurately predict the shock (unpredictable) on a given trial; however, in both cases the auditory cue still serves as a better predictor of the UCS than contextual information present during training. This allows us to determine whether the predictability of the aversive event by the auditory cue is what drives reconsolidation following retrieval. In addition, in the previous experiment we found that the hippocampus was engaged during the reconsolidation of an auditory fear memory, which is largely a hippocampus-independent task.
As the hippocampus responds to contextual information, it is possible that new contextual information present during retrieval initiates the reconsolidation process. To account for this, in some animals we eliminated new contextual information during retrieval by pre-exposing them to the retrieval conditions the day prior to training. This allowed us to determine whether new contextual information is incorporated in the memory trace and becomes a predictor of the aversive event.

During the training session we found a main effect for time $\left(F_{(1,61)}=2988.493, P<0.001\right)$ and condition $\left(F_{(4,61)}=7.430\right.$, $P<0.001)$ and a time by condition interaction $\left(F_{(4,61)}=3.663\right.$, $P<0.05$; Figure 2b). As might be expected with the unpredictable condition, animals that received $50 \%$ reinforcement during training showed more fear behavior during the training session. However, during the retrieval session we found a main effect for time $\left(F_{(1,49)}=195.351, P<0.001\right)$ but not condition $\left(F_{(3,49)}=0.066, P=0.978\right)$ and there was not a time by condition interaction $\left(F_{(3,49)}=1.559, \quad P=0.211\right.$; Figure $2 c$ ), suggesting that the different training parameters did not alter the strength of the memory for the auditory cue.

We next examined changes in proteasome activity and AMPAR subunit expression as markers of memory reconsolidation. Surprisingly, we found that eliminating new contextual information, but not the ability of the auditory cue to predict the aversive stimulus, completely prevented the increases in proteasome activity $\left(F_{(4,57)}=2.622, P<0.05\right.$; Figure $3 \mathrm{a})$ and decreases in GluR2 $\left(F_{(4,52)}=3.903, P<0.01\right.$; Figure $3 \mathrm{~b}$ ) expression in the amygdala following retrieval without altering $\beta$-actin expression $\left(F_{(4,58)}=0.239, P=0.915\right.$; Figure $3 b)$. However, we did not find a main effect for GluR1 expression $\left(F_{(4,54)}=1.219, P=0.314\right.$; Figure $\left.3 \mathrm{~b}\right)$, as most 
a

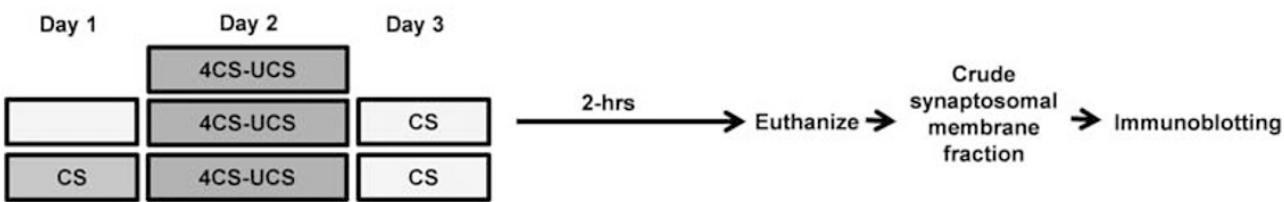

b

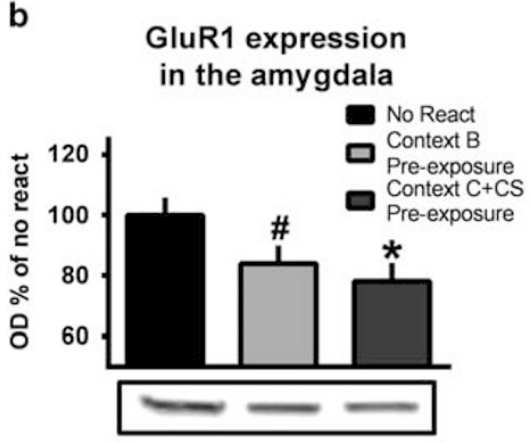

d

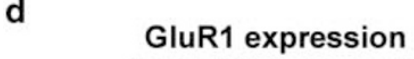
in the hippocampus

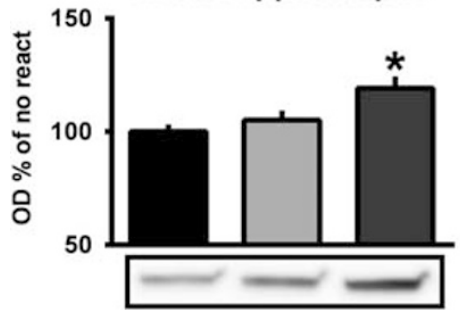

GluR2 expression in the amygdala

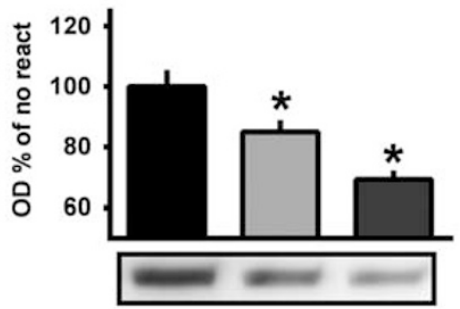

GluR2 expression in the hippocampus

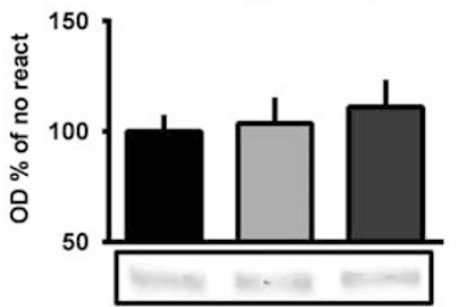

C

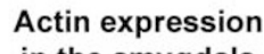
in the amygdala

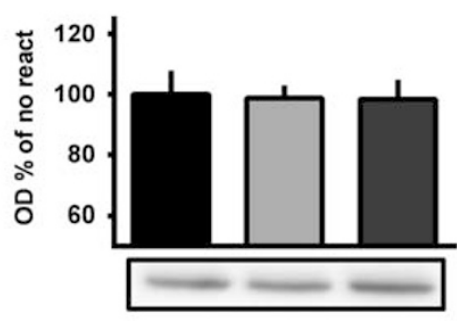

e

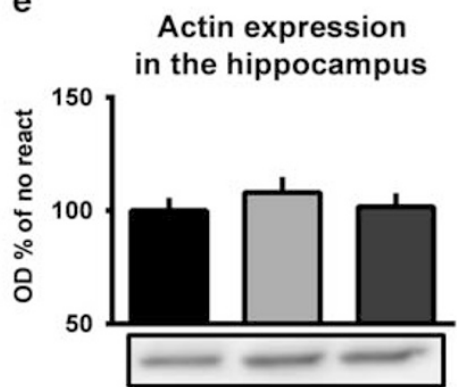

Figure 4 Pre-exposing animals to the retrieval context or auditory cue by themselves do prevent memory reconsolidation following retrieval. (a) Rats were trained to 100\% reinforcement auditory fear conditioning with pre-exposure to the retrieval context or the auditory cue in Context $C$ the day before training. All animals received a retrieval in Context B on day 3 and amygdala tissue collected 120 min later $(n=1 \mid-12$ per group). (b) Pre-exposure to the retrieval context or the CS in Context C by themselves decreased GluRI and GluR2 expression in the amygdala after retrieval without altering $\beta$-actin expression (c). (d) Pre-exposing animals to the retrieval context, but not the auditory cue in Context C, prevented increases in GluRI expression in the hippocampus following retrieval without altering GluR2 or b-actin expression (e). $* P<0.05$ from no retrieval. $\# P=0.07$ from no retrieval.

groups showed a similar pattern of moderately reduced GluR1 expression in the amygdala following retrieval, suggesting that in regard to AMPAR subunit expression, changes in GluR2 is the best marker of memory destabilization in the amygdala (Hong et al, 2013). In addition, a pairwise $t$-test revealed that pre-exposing animals to the retrieval conditions also prevented increases in GluR1 expression in the hippocampus following retrieval $\left(t_{(58)}=\right.$ 2.291, $P<0.05$; Figure 3d) without altering proteasome activity $\left(F_{(4,57)}=0.368, P=0.818\right.$; Figure $\left.3 \mathrm{c}\right)$ or $\beta$-actin expression $\left(F_{(4,57)}=0.223, P=0.925\right.$; Figure $\left.3 \mathrm{~d}\right)$. Surprisingly, we found a trend for a main effect in GluR2 expression in the hippocampus $\left(F_{(4,58)}=2.182, P=0.082\right.$; Figure $\left.3 \mathrm{~d}\right)$. Pairwise $t$-tests revealed that although retrieval of an auditory fear memory does not normally alter GluR2 expression in the hippocampus $\left(t_{(21)}=-0.678, P=0.505\right)$, either pre-exposing animals to the retrieval conditions $\left(t_{(23)}=-2.335, P=0.029\right)$ or training them to $50 \%$ reinforcement $\left(t_{(23)}=-2.101\right.$, $P=0.047)$ results in increased GluR2 expression in this region. These surprising changes in GluR1/2 expression suggest that the differential training procedures may have altered synaptic potentiation in the hippocampus following memory retrieval. Collectively, these results suggest that new contextual information present during retrieval drives the reconsolidation-dependent updating for both predictable and unpredictable fear associations, indicating that new contextual information present during retrieval is incorporated into the memory trace.

To exclude the possibility that alterations in the reconsolidation process in the retrieval pre-exposure condition were a result of pre-exposure to the auditory cue or context alone, we examined changes in AMPAR subunit expression in the amygdala following retrieval under conditions in which animals were pre-exposed to the retrieval context or the auditory cue in a novel context prior to training (Figure $4 \mathrm{a}$ ), as GluR1/2 expression was the only mechanism to be altered in both the amygdala and hippocampus in the previous experiments. We found a reduction in GluR1 $\left(F_{(2,29)}=3.503\right.$, $P=0.043$; Figure $4 \mathrm{~b})$ and GluR2 expression $\left(F_{(2,28)}=12.148\right.$, $P<0.001$; Figure $4 \mathrm{~b})$ in both the context exposure and auditory cue exposure groups without any changes in $\beta$-actin expression $\left(F_{(2,26)}=0.016, P=0.984\right.$; Figure $\left.4 \mathrm{c}\right)$, suggesting that either pre-exposing animals to the retrieval context alone or the auditory cue in a novel context not used for training or retrieval did not prevent the normal retrieval-dependent reductions in AMPAR subunit expression in the amygdala. However, GluR1 levels were reduced in the hippocampus by just exposing animals to the retrieval context $\left(F_{(2,29)}=3.503\right.$, $P<0.05$; Figure $4 \mathrm{~d})$, though GluR2 $\left(F_{(2,29)}=0.253, P=0.778\right.$; Figure $4 \mathrm{~d})$ and $\beta$-actin expression $\left(F_{(2,30)}=0.425, P=0.658\right.$; 
a

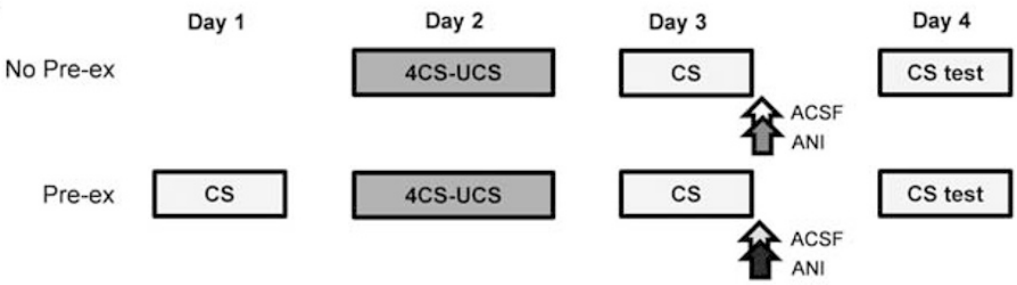

b

C
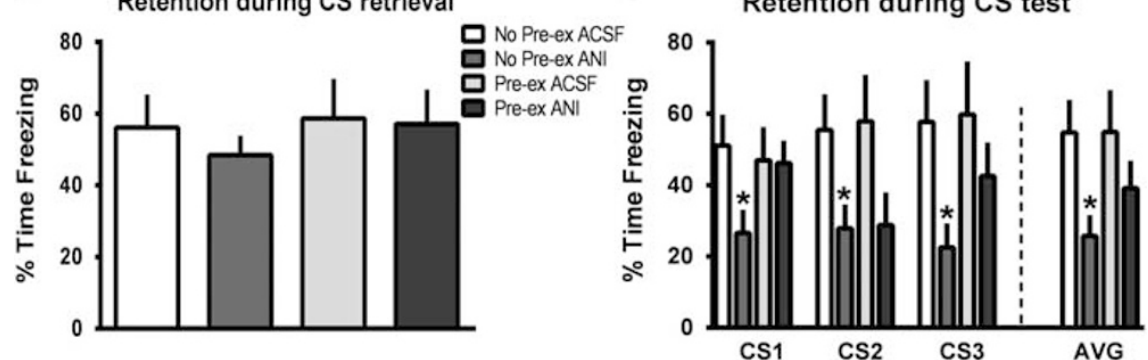

Figure 5 Pre-exposing animals to the retrieval conditions prevents memory impairments from post-retrieval protein synthesis inhibition in the amygdala. (a) Rats were trained with 100\% contingent auditory fear conditioning with or without 'retrieval pre-exposure' the day before training. On the day after training, animals received a brief retrieval followed by infusions of vehicle or the protein synthesis inhibitor anisomycin (ANI) into the amygdala. All groups were tested for memory with the auditory cue $24 \mathrm{~h}$ after the retrieval/infusion procedure ( $n=7-8$ per group). (b) There were no differences between groups during retrieval. (c) The group that received ANI without retrieval pre-exposure had significant impairments in memory for the auditory cue relative to controls, whereas the groups receiving $\mathrm{ANI}$ with retrieval pre-exposure performed similar to controls throughout most of the test session. $* P<0.05$ from ACSF.

Figure 4e) remained unchanged, suggesting that the alterations we observed in the reconsolidation process in the amygdala in the previous experiment were specifically due to the pairing of the auditory cue with the retrieval context during pre-exposure, while the hippocampus was responding specially to the novelty of the context.

Our biochemical data suggest that pre-exposing animals to the retrieval conditions prevent the reconsolidation process from occurring. To test this directly, we assessed whether a retrieved memory was susceptible to post-retrieval blockade of protein synthesis in the amygdala following our retrieval preexposure procedure (eg, Ben Mamou et al, 2006; Jarome et al, 2011). Animals were implanted with chronic cannula aimed at the amygdala and underwent the retrieval pre-exposure as described above or did not receive the pre-exposure (Figure 5a). All animals were then trained to auditory fear conditioning and received a brief retrieval the following day, followed immediately by infusions of vehicle or the protein synthesis inhibitor ANI into the amygdala, and tested the next day. During retrieval there were no differences between groups (Figure $5 b$ ); however, there were significant differences between groups during the test session (Figure 5c). Although on average the group that did not receive pre-exposure had significant impairments in memory for the auditory cue relative to controls $\left(t_{(13)}=2.561, P<0.05\right)$, this was not evident in pre-exposure group $\left(t_{(14)}=1.113, P=0.284\right)$. In addition, although the no pre-exposure group had impaired memory for the CS relative to controls at all three CS presentations (all $P$ 's $<0.05)$, the pre-exposure group had no impairments relative to controls at the first or third CS presentation (all $P$ 's $>0.05)$, with only a trend at the second CS $(P=0.09)$. Furthermore, at the first CS presentation the pre-exposure animals receiving ANI had significantly higher memory retention than did the no pre-exposure animals receiving ANI $\left(t_{(13)}=2.151, P=0.05\right)$. Collectively, this suggests that pre-exposing animals to the retrieval conditions largely prevented the effectiveness of ANI at disrupting the reconsolidation process, supporting our biochemical data. This indicates that during retrieval, the fear-provoking auditory cue became associated with the context, suggesting that reconsolidation was largely triggered by the incorporation of new contextual information into the memory trace.

\section{DISCUSSION}

The traumatic memories that underlie a variety of anxiety disorders are often difficult to treat and can remain present even following extensive behavioral intervention. Reconsolidation has emerged as a potentially important target for the treatment of some disorders as it provides the unique opportunity to 'update' or 'modify' maladaptive memories. However, it currently remains unknown why memories undergo reconsolidation following retrieval. Such information is critical to properly design potential therapeutic treatments using reconsolidation principles. In the present study we asked what new information is present at the time of retrieval that drives the reconsolidation of an auditory fear memory in rodents. By systematically manipulating the animals training experience, we found that pre-exposing animals to the context-CS association present during retrieval prevented changes in proteasome activity and GluR2 expression in the amygdala, and prevented the susceptibility of the retrieved memory to post-retrieval protein synthesis blockade, suggesting that the reconsolidation process was not initiated. These results demonstrate for the first time that new contextual associations are learned during retrieval and incorporated into the memory trace by reconsolidation, suggesting that controlling new contextual information during reconsolidation-based treatments of 
anxiety disorders associated with traumatic memories should be a critical focus moving forward.

Although the molecular mechanisms underlying the reconsolidation process have only begun to be elucidated, a number of recent studies have focused on changes in ubiquitin-proteasome activity and AMPA receptor subunit exchange as potential regulators of memory reconsolidation (reviewed in, Jarome and Helmstetter, 2013a). For example, inhibition of proteasome activity prevents the initiation of the reconsolidation process (Lee et al, 2008; Jarome et al, 2011) and can prevent reconsolidation-dependent memory strengthening (Lee, 2008). In addition, reconsolidation requires dynamic changes in AMPA receptor subunit expression, particularly the exchange of GluR2 containing AMPA receptors for those lacking GluR2 (Rao-Ruiz et al, 2011; Hong et al, 2013), an effect dependent on GluR1 phosphorylation (Clem and Huganir, 2010). Thus, changes in proteasome activity and GluR2 expression levels are critical regulators of the reconsolidation process. In the present study, we found that pre-exposing animals to the retrieval conditions prevented increases in proteasome activity and decreases in GluR2 expression at amygdala synapses. This suggests that the reconsolidation process did not occur, and provides support for the idea that reconsolidation and its associated mechanisms occur specifically in response to new information present during the retrieval session.

One surprising finding from our study was that under our conditions new contextual information had a greater influence on the ability of the retrieved memory to undergo reconsolidation than did the predictive cue present during the learning of the aversive association. This result contradicts the theory that 'prediction error' governs the reconsolidation of memories following retrieval (Diaz-Mataix et al, 2013; Sevenster et al, 2013). One potential reason for this conflicting finding is that it is possible that following a single CS presentation, no new information can be learned about the predictability of the aversive stimulus's occurrence when only the cue is present. Thus, for the cue to provide any new information during retrieval, the aversive stimulus it was originally associated with must be present and in the absence of this aversive stimulus, contextual cues may be the next best predictor of CS occurrence. For this reason the new contextual information becomes associated with the now aversive cue. In addition, it is possible that during retrieval the context becomes an 'occasion setter', signaling that the auditory cue will not be followed by the aversive stimulus (Urcelay and Miller, 2014). This interpretation would predict that the memory would not undergo reconsolidation following each subsequent retrieval, as the occasion has been set during the first retrieval. However, we previously found that a memory undergoes reconsolidation even after multiple retrieval events, as long as the retrieval events are temporally separated (Jarome et al, 2012), suggesting that this interpretation cannot explain our present results. Collectively, these results suggest that it is possible that new contextual information present during retrieval may be a better predictor of the aversive event than the cues originally associated with that event, though it is unclear exactly why this occurs.

If contextual information is incorporated into the memory trace following retrieval then this contextual information should become a predictor of the aversive stimulus. One possible explanation for how this occurs is through higher- order conditioning where the contextual information becomes associated with the cue that predicts the UCS (Helmstetter and Fanselow, 1989). In this case, repeated presentations of a CS in a new context could result in an association between the aversive cue and the neutral context. Through this association, the contextual cue could acquire the ability to predict the occurrence of the aversive stimulus without the original cue being present. Although there is some evidence that associations learned through higherorder conditioning do undergo reconsolidation (Debiec et al, 2006), few studies have systematically tested if second-order conditioning requires mechanisms similar to that of reconsolidation and if this form of conditioning is 'updating' or 'new learning'. Thus, whether reconsolidation 'updates' memories through a process similar to higher-order conditioning will be of interest in future studies.

Although our data strongly suggest that contextual information drives the initiation of the reconsolidation process, we cannot completely rule out the potential influence of prediction error. For example, it is possible that during the retrieval pre-exposure condition (Figure 3) animals learned to predict that the CS will not be followed by shock in this context. However, this interpretation would require the animal to make a prediction based on cumulative information from both the pre-exposure and training sessions, as the shock was not introduced prior to pre-exposure. In addition, prediction error could explain why pre-exposing animals to the CS in a novel context not used for training or retrieval can still result in the initiation of reconsolidation mechanisms (Figure 4), as the animals prediction about the CS-UCS relationship could continually change across the training days. However, as the only difference between the above-mentioned procedures (Figures 3 and 4) is whether the retrieval context is used for pre-exposure, it seems likely that contextual information has a strong influence on initiation of the reconsolidation process, though it is possible that both contextual information and prediction error are necessary for reconsolidation to occur. Future studies will need to directly test the exact conditions under which prediction error and contextual information drive the initiation of the reconsolidation process.

In conclusion, we found that eliminating new contextual information during retrieval prevents the reconsolidation of both predictable and unpredictable fear associations. In addition, these results suggest that the auditory cue becomes associated with the context during reconsolidation, indicating that under normal conditions animals are incorporating new information into the memory trace during reconsolidation. These results suggest that targeting of new contextual information present during retrieval may be an effective strategy for the treatment of traumatic memories using memory reconsolidation.

\section{FUNDING AND DISCLOSURE}

The authors declare no conflict of interest.

\section{ACKNOWLEDGMENTS}

This work was supported by National Institute of Mental Health grants R01-06558 (FJH) and F31-088125 (TJJ) and the American Psychological Foundation (TJJ). 


\section{REFERENCES}

Alberini CM, Chen DY (2012). Memory enhancement: consolidation, reconsolidation and insulin-like growth factor 2. Trends Neurosci 35: 274-283.

Alberini CM, Ledoux JE (2013). Memory reconsolidation. Curr Biol 23: R746-R750.

Ben Mamou C, Gamache K, Nader K (2006). NMDA receptors are critical for unleashing consolidated auditory fear memories. Nat Neurosci 9: 1237-1239.

Clem RL, Huganir RL (2010). Calcium-permeable AMPA receptor dynamics mediate fear memory erasure. Science 330: 1108-1112.

Debiec J, Doyere V, Nader K, Ledoux JE (2006). Directly reactivated, but not indirectly reactivated, memories undergo reconsolidation in the amygdala. Proc Natl Acad Sci USA 103: 3428-3433.

Diaz-Mataix L, Ruiz Martinez RC, Schafe GE, Ledoux JE, Doyere V (2013). Detection of a temporal error triggers reconsolidation of amygdala-dependent memories. Curr Biol 23: 467-472.

Finnie PS, Nader K (2012). The role of metaplasticity mechanisms in regulating memory destabilization and reconsolidation. Neurosci Biobehav Rev 36: 1667-1707.

Gilmartin MR, Kwapis JL, Helmstetter FJ (2013a). NR2A- and NR2B-containing NMDA receptors in the prelimbic medial prefrontal cortex differentially mediate trace, delay, and contextual fear conditioning. Learn Mem 20: 290-294.

Gilmartin MR, Miyawaki H, Helmstetter FJ, Diba K (2013b). Prefrontal activity links nonoverlapping events in memory. J Neurosci 33: 10910-10914.

Helmstetter FJ, Fanselow MS (1989). Differential second-order aversive conditioning using contextual stimuli. Anim Learn Behav 17: 205-212.

Hong I, Kim J, Lee S, Ko HG, Nader K, Kaang BK et al (2013). AMPA receptor exchange underlies transient memory destabilization on retrieval. Proc Natl Acad Sci USA 110: 8218-8223.

Inda MC, Muravieva EV, Alberini CM (2011). Memory retrieval and the passage of time: from reconsolidation and strengthening to extinction. J Neurosci 31: 1635-1643.

Jarome TJ, Helmstetter FJ (2013a). The ubiquitin-proteasome system as a critical regulator of synaptic plasticity and longterm memory formation. Neurobiol Learn Mem 105: 107-116.

Jarome TJ, Kwapis JL, Ruenzel WL, Helmstetter FJ (2013b). CaMKII, but not protein kinase A, regulates Rpt6 phosphorylation and proteasome activity during the formation of long-term memories. Front Behav Neurosci 7: 115.

Jarome TJ, Kwapis JL, Werner CT, Parsons RG, Gafford GM, Helmstetter FJ (2012). The timing of multiple retrieval events can alter GluR1 phosphorylation and the requirement for protein synthesis in fear memory reconsolidation. Learn Mem 19: 300-306.

Jarome TJ, Werner CT, Kwapis JL, Helmstetter FJ (2011). Activity dependent protein degradation is critical for the formation and stability of fear memory in the amygdala. PloS One 6: e24349.

Kwapis JL, Jarome TJ, Schiff JC, Helmstetter FJ (2011). Memory consolidation in both trace and delay fear conditioning is disrupted by intra-amygdala infusion of the protein synthesis inhibitor anisomycin. Learn Mem 18: 728-732.

Lee JL (2008). Memory reconsolidation mediates the strengthening of memories by additional learning. Nat Neurosci 11: 1264-1266.

Lee SH, Choi JH, Lee N, Lee HR, Kim JI, Yu NK et al (2008). Synaptic protein degradation underlies destabilization of retrieved fear memory. Science 319: 1253-1256.

Monfils MH, Cowansage KK, Klann E, LeDoux JE (2009). Extinction-reconsolidation boundaries: key to persistent attenuation of fear memories. Science 324: 951-955.

Nader K, Schafe GE, Le Doux JE (2000). Fear memories require protein synthesis in the amygdala for reconsolidation after retrieval. Nature 406: 722-726.

Parsons RG, Gafford GM, Helmstetter FJ (2006). Translational control via the mammalian target of rapamycin pathway is critical for the formation and stability of long-term fear memory in amygdala neurons. J Neurosci 26: 12977-12983.

Parsons RG, Ressler KJ (2013). Implications of memory modulation for post-traumatic stress and fear disorders. Nat Neurosci 16: 146-153.

Pavlov IP (1927). Conditioned Reflexes: an Investigation of the Physiological Activity of the Cerebral Cortex. London, London: Oxford University Press.

Rao-Ruiz P, Rotaru DC, van der Loo RJ, Mansvelder HD, Stiedl O, Smit AB et al (2011). Retrieval-specific endocytosis of GluA2AMPARs underlies adaptive reconsolidation of contextual fear. Nat Neurosci 14: 1302-1308.

Schiller D, Monfils MH, Raio CM, Johnson DC, Ledoux JE, Phelps EA (2010). Preventing the return of fear in humans using reconsolidation update mechanisms. Nature 463: 49-53.

Sevenster D, Beckers T, Kindt M (2013). Prediction error governs pharmacologically induced amnesia for learned fear. Science 339: $830-833$.

Tronson NC, Wiseman SL, Olausson P, Taylor JR (2006). Bidirectional behavioral plasticity of memory reconsolidation depends on amygdalar protein kinase A. Nat Neurosci 9: 167-169.

Urcelay GP, Miller RR (2014). The functions of contexts in associative learning. Behav Processes 104: 2-12. 